\title{
STEADY STATES OF THE REACTION-DIFFUSION EQUATIONS. PART I: QUESTIONS OF EXISTENCE AND CONTINUITY OF SOLUTION BRANCHES
}

\author{
J. G. BURNELL', A. A. LACEY $Y^{1,2}$ and G. C. WAKE ${ }^{1}$
}

(Received 24 May 1982)

\begin{abstract}
When material is undergoing an exothermic chemical reaction which is sustained by the diffusion of a reactant, the steady-state regime is governed by a coupled pair of nonlinear elliptic partial differential equations with linear boundary conditions. In this paper we consider questions of existence of solutions to these equations. It is shown that, with the exception of the special case in which the mass-transfer is uninhibited on the boundary, a solution always exists, whereas in this special case a solution exists only for sufficiently low values of the exothermicity. Bounds are established for the solutions and the occurrence of minimal and maximal solutions is shown for some cases. Finally the behaviour of the solution set with respect to one of the parameters is studied.
\end{abstract}

\section{Introduction}

Consider a first-order exothermic reaction taking place within a porous medium where we assume that the concentration of just one diffusing reactant, together with temperature, controls the rate of reaction. Any convection effects, forced or natural, are neglected. Taking the Arrhenius reaction rate law, this system can be modelled by the coupled parabolic equations

$$
\begin{gathered}
C_{p} \frac{\partial T}{\partial \hat{t}}=\phi H A c \exp (-E / R T)+k \nabla^{2} T, \\
\frac{\partial c}{\partial \hat{t}}=-A c \exp (-E / R T)+D \nabla^{2} T,
\end{gathered}
$$

\footnotetext{
'Mathematics Department, Victoria University, Private Bag, Wellington, New Zealand. Please address all correspondence to Dr. Wake at this address.

${ }^{2}$ Present address: Mathematics Department, Heriot-Watt University, Edinburgh EH14 4AS, Scotland. (C) Copyright Australian Mathematical Society 1983
} 
in the region $\hat{\Omega}$ and $\hat{t}>0$. Here $\hat{t}$ is time, $c$ is the concentration of the diffusing reactant in pores, $C_{p}$ is the volumetric specific heat, $E$ is the activation energy of the reaction, $R$ is the universal gas constant, $T$ is the local temperature, $\phi$ is the porosity of the medium, $H$ is the exothermicity per unit volume, $A$ is a rate constant (frequency factor), $k$ is the thermal conductivity and $D$ is the diffusivity of the reactant $c$. We shall assume that all these quantities are constant except that $c$ and $T$ depend on position and $\hat{t}$. Also we note that the values of $C_{p}, A, k$ are effective values with specific heat and thermal conductivity taking different values in the matrix and the fluid-occupied spaces, while the reaction occurs only on the interfaces between the solid matrix and the fluid. Equations of the form of (1) are derived in Aris [2, Chapter 1].

The reacting region $\hat{\Omega}$ is assumed to be surrounded by an infinite reservoir containing a fixed concentration $c_{a}$ of the controlling reactant and which is maintained at a constant ambient temperature $T_{a}$. The boundary conditions on the surface $\partial \hat{\Omega}$ are

$$
\begin{array}{lll}
\frac{\partial T}{\partial n} \propto\left(T-T_{a}\right) & \text { or } & T=T_{a}, \\
\frac{\partial c}{\partial n} \propto\left(c-c_{a}\right) & \text { or } & c=c_{a} .
\end{array}
$$

Such a system arises from the diffusion of oxygen into a tightly packed material such as wool or coal, with $c$ being the concentration of oxygen; or the diffusion of some nutrient with concentration $c$ into a biological cell.

The systems of equations (1), (2) can be nondimensionalised to yield the approximate model

$$
\begin{gathered}
\partial u / \partial t=\nabla^{2} u+\lambda(1+v) \exp u \text { in } \Omega, \quad t>0, \\
L(\partial v / \partial t)=\nabla^{2} v-\alpha \lambda(1+v) \exp u \text { in } \Omega, \quad t>0, \\
\partial u / \partial n+\mu u=0 \text { on } \partial \Omega, \\
\partial v / \partial n+\nu v=0 \text { on } \partial \Omega .
\end{gathered}
$$

The equations (3a), (3b) are approximations to the true model because of the Frank-Kamenetskii approximation

$$
\exp \left(-\frac{E}{R T}\right)=\exp \left(-\frac{E}{R T_{a}}\right) \exp \left(\frac{u}{1+\varepsilon u}\right) \approx \exp \left(-\frac{E}{R T_{a}}\right) \exp u,
$$

where $u=E\left(T-T_{a}\right) / R T_{a}^{2}$ is a dimensionless temperature excess and $\varepsilon=$ $R T_{a} / E \ll 1$. Also we have $v=c / c_{a}-1$ is a dimensionless reactant concentration, $t=k \hat{t} / d^{2} C_{p}$ is a dimensionless time, $d$ is a characteristic length of $\hat{\Omega}$ such as half of the diameter, $L=k / D C_{p}$ is the Lewis number,

$$
\lambda=\phi E d^{2} H c_{a} \exp \left(-E / R T_{a}\right) / k R T_{a}^{2}
$$


is the Frank-Kamenetskii parameter, $\alpha=k R T_{a}^{2} / H D E c_{a}$, $\mu$ is the heat-transfer coefficient (Biot number) on the boundary, $\nu$ is the mass-transfer coefficient on the boundary, and $\Omega$ is the region $\hat{\Omega}$ in the nondimensional spatial coordinates. In this paper we shall assume that the constants $\lambda, \alpha, \mu, \nu$ are positive real numbers or satisfy the following special cases:

(i) $\lambda=0$, that is, no heat produced $(H=0)$;

(ii) $\alpha=0$, that is, infinite diffusion of reactant $(D=\infty)$ in which case $v \equiv 0$ and the system (3) reduces to just one equation, the classical case;

(iii) $\mu=\infty$, that is, infinite surface heat-transfer in which case the boundary condition (4a) becomes the Dirichlet condition $u=0$;

(iv) $\nu=\infty$, that is, infinite surface mass-transfer with the condition (4b) becoming $v=0$.

Our purpose here is to investigate the steady-states of the system (3) and related systems ( shall investigate conditions under which such steady-states exist and, if so, how many steady solutions do exist. These questions are related to the question of 'critical behaviour' of these systems where large discontinuous changes in the steady-state regime occur for small changes in the parameters. The steady system of (3) is

$$
\begin{array}{cc}
\nabla^{2} u+\lambda(1+v) \exp u=0 & \text { in } \Omega, \\
\nabla^{2} v-\alpha \lambda(1+v) \exp u=0 & \text { in } \Omega,
\end{array}
$$

with the boundary conditions (4) holding on the boundary $\partial \Omega$.

A primary motivation of this study occurs from the special case of $\alpha=0$ in which case $v \equiv 0$ and so (3) reduces to

$$
\partial u / \partial t=\nabla^{2} u+\lambda e^{u} \text { in } \Omega,
$$

with the condition (4a) on the boundary. It has been found previously (Keller and Cohen [6], Fujita [4]) that there exists a value of $\lambda$, say $\lambda^{*}$, such that there is a steady solution of (6), (4a) for $\lambda<\lambda^{*}$ but not for $\lambda>\lambda^{*}$. (We then say $\left(0, \lambda^{*}\right)$ is the spectrum of the problem.) Moreover for $\lambda<\lambda^{*}$ if the initial condition is small enough then $u$ tends to one of the steady-state solutions (Fujita [5], Sattinger [11]), while for $\lambda>\lambda^{*}, u$ "blows-up", that is, $u$ becomes infinite in either infinite or finite time (Fujita [4], Lacey [7]). It is clear that for, this special case at least, there is a "critical" value of $\lambda$, namely $\lambda^{*}$.

We shall consider the more general problem (4), (5) to determine if there is a similar number $\lambda^{*}$, that is, a value such that a solution to the equations exists if $\lambda<\lambda^{*}$, but not for $\lambda>\lambda^{*}$. It transpires that for $\alpha>0$ there is one special case of which a finite value of $\lambda^{*}$ exists, but for all other cases a solution to equations (4), (5) exists for all values of $\lambda$. Somewhat similar to the quantity $\lambda^{*}$ is a value $\lambda_{\mathrm{cr}}$ (a critical value) of $\lambda$ at which there is a turning point in the $((u, v), \lambda)$ bifurcation 
diagram. At this point there may be a jump in the minimal solution (if such a solution exists) as $\lambda$ passes through $\lambda_{\mathrm{cr}}$. This change in the solution to (4), (5) is liable to correspond to a sudden alteration to the behaviour of the parabolic equation. Again this is suggested by the behaviour of the solutions of the single time-dependent equation (6) which approach the minimal steady-state solution if the initial data is small enough (Sattinger [11]).

We shall frequently replace in (5) the specific function $(1+v) e^{u}$ with a more general function $f$, defined on $\mathbf{R}^{2}$, which is required to satisfy:

(i) there is some $v_{0}<0$ such that $f\left(u, v_{0}\right)=0$ for all $u \in \mathbf{R}$;

(ii) $f(u, \cdot): y \mapsto f(u, y)$ and $f(\cdot, v): x \mapsto f(x, v)$ are monotonic increasing for all $u \in \mathbf{R}$ and $v \geqslant v_{0}$ respectively;

(iii) $f \in C^{1}\left(\mathbf{R}^{2}\right)$.

Accordingly, (5) is then replaced by the system

$$
\begin{gathered}
\nabla^{2} u+\lambda f(u, v)=0 \quad \text { in } \Omega, \\
\nabla^{2} v-\lambda \alpha f(u, v)=0 \quad \text { in } \Omega,
\end{gathered}
$$

together with the same boundary conditions (4) on $\partial \Omega$. The above case covers the situation when the Frank-Kamenetskii approximation is not made $(\varepsilon \neq 0)$ in equations (3) and the exact formulation of equations (1) in dimensionless coordinates has

$$
f(u, v)=(1+v) \exp (u /(1+\varepsilon u)) .
$$

We shall frequently have occasion to use alternative formulations. In particular, if we let

$$
h=\alpha u+v
$$

and substitute for either $u$ in terms of $v$ and $h$ or $v$ in terms of $u$ and $h$, we obtain

$$
\left.\begin{array}{cc}
\nabla^{2} v-\lambda \alpha f((h-v) / \alpha, v)=0 & \text { in } \Omega, \\
\frac{\partial v}{\partial n}+\nu v=0 & \text { on } \partial \Omega,
\end{array}\right\},
$$

or

$$
\left.\begin{array}{cc}
\nabla^{2} u+\lambda f(u, h-\alpha u)=0 & \text { in } \Omega, \\
\frac{\partial u}{\partial n}+\mu u=0 & \text { on } \partial \Omega,
\end{array}\right\}
$$


We note that in these forms of the problem, (10) and (11), the boundary conditions for $h$ change if $\mu$ or $\nu$ is infinite. In (10b) if $\mu=\infty>\nu$ we have $h=\alpha u$ on $\partial \Omega$; if $\nu=\infty>\mu$ we have $h=\nu$ on $\partial \Omega$; in both (10b) and (11b), and so $h=0$ in $\bar{\Omega}$.

Throughout this paper we shall take $\Omega$ as an open bounded subset $\mathbf{R}^{n}$ (usually $n=3$ ) with a smooth boundary $\partial \Omega$. In particular we require that $\partial \Omega$ is an $n-1$ dimensional $C^{2+\sigma}$ manifold, where $0 \leqslant \sigma<1$. This assumption gives the property that $u \in C^{k}(\bar{\Omega})$ implies $u \in C^{k-1+\beta}(\bar{\Omega})$, for any $0<\beta<1$.

We now proceed to the main results concerning existence of solutions.

\section{Existence of solutions}

We shall show in this section that if $\nu<\infty$ or if $\mu=\nu=\infty$, then solutions to the problem (4), (7) and of course (4), (5) exist for all values of $\lambda$ provided that $\alpha>0$. We shall use the theory of topological degree to prove this.

The following three results which are stated in Amann [1] and are proved in Ladyzhenskaya and Ural'tseva [8] (Proposition 1), and Lloyd [9] (Propositions 2,3) will be used:

Proposition 1. Let $\Omega$ be as in Section 1. Then for each $g \in C^{\sigma}(\bar{\Omega})$ there is an element in $C^{2+\sigma}(\bar{\Omega}), K_{\nu} g$ say, which is the unique solution of

$$
-\nabla^{2} w=g \quad \text { in } \Omega ; \quad \partial w / \partial n+\nu w=0 \quad \text { on } \partial \Omega .
$$

Furthermore the map $K_{\nu}: C^{\sigma}(\bar{\Omega}) \rightarrow C^{2+o}(\bar{\Omega}): g \mapsto K_{\nu} g$ is a positive linear operator which can be extended uniquely to a compact linear operator, also denoted by $K_{\nu}$, from $C(\bar{\Omega})$ into $C^{\beta}(\bar{\Omega})$ for any $0 \leqslant \beta<2$ (where $C^{\beta}(\bar{\Omega})$ has its usual norm).

(The solution operators $K_{\nu}$ are just the linear integral operators with kernel the Green's function for $-\nabla^{2}$ with the appropriate boundary condition.)

Proposition 2. Suppose $X$ is a normed vector space and $D$ is an open, bounded subset of $X$ containing 0 . Further, suppose $T: \bar{D} \rightarrow X$ is a compact map (that is, it is continuous and maps bounded sets into relatively compact sets) such that $0 \notin$ $(I-T)(\partial D)$. Then an integer $d(I-T, D, 0)$, called the degree of $I-T$ at 0 on $D$, is defined and the following hold:

(i) if $T=0$ then $d(I, D, 0)=1$;

(ii) if $d(I-T, D, 0) \neq 0$ then there exists $x \in D$ such that $x-T x=0$;

(iii) if $H: \bar{D} \times[0,1] \rightarrow X$ satisfies:

(a) $(\forall s \in[0,1]) H(\cdot, s): \bar{D} \rightarrow X: x \mapsto H(x, s)$ is compact. 
(b) $(\forall \varepsilon>0)(\exists \delta>0)$ such that whenever $\left|s_{1}-s_{2}\right|<\delta$ we have

$$
(\forall x \in \bar{D})\left\|H\left(x, s_{1}\right)-H\left(x, s_{2}\right)\right\|<\varepsilon \text {, and }
$$

(c) $(\forall s \in[0,1]) 0 \notin(I-H(\cdot, s))(\partial D)$, then $d(I-H(\cdot, s), D, 0)$ is defined for all $s \in[0,1]$, and is independent of $s$.

Further, we have a slightly more general version of (iii) which we shall make use of:

(iv) if $U$ is an open bounded subset of $X \times[0,1]$ and $H: \bar{U} \rightarrow X$ is a compact map such that $0 \notin(I-H)(\partial U)$ then $d\left(I-H(\cdot, s), U_{s}, 0\right)$ is defined for each $s \in[0,1]$, and is independent of $s$, where

$$
U_{s}=\{x:(x, s) \in U\} .
$$

Proposition 3. Suppose $T$ and $D$ are as in Proposition 2.

(i) (Excision Property) If $E \subseteq \bar{D}$ is closed and $0 \notin(I-T)(E)$ then

$$
d(I-T, D, 0)=d(I-T, D \backslash E, 0) .
$$

(ii) There exists $\delta>0$ (depending on $T$ and $D$ ) such that if $S$ is a compact map on $\bar{D}$ with $(\forall x \in \bar{D})\|T(x)-S(x)\|<\delta$ then $d(I-S, D, 0)$ is defined and equals $d(I-T, D, 0)$.

We shall demonstrate the existence of a solution to the problem (4), (7) by showing the equivalence of solutions $(u, v)$ to $(4),(7)$ and fixed points of a certain map $T$. We then use degree theory (Propositions 2,3 ) to show the existence of fixed points of $T$, and hence solutions to the system (4), (7).

We define a map $T$ on $C(\bar{\Omega}) \times C(\bar{\Omega})$ by

$$
T(u, v)=\left(\lambda K_{\mu} F(u, v),-\alpha K_{\nu} F(u, v)\right)
$$

where $K_{\mu}, K_{\nu}$ were defined in Proposition 1 and $F(u, v)$ is the Nemystsky operator given by

$$
F(u, v)(x)=f(u(x), v(x)) .
$$

We now establish the necessary properties of $T$ which ensure it has a fixed point.

Proposition 4. $T$ is a compact map from $C(\bar{\Omega}) \times C(\bar{\Omega})$ into itself (using the supremum norm on $C(\bar{\Omega})$ ).

Proof. By Proposition $1, K_{\mu}, K_{\nu}$ are compact in the supremum norm on $C(\bar{\Omega})$ and map into $C(\bar{\Omega})$. So it suffices to show that the map $(u, v) \mapsto F(u, v)$ is continuous and bounded. This follows from the requirement, (iii) in Section 1, that $f \in C^{1}\left(\mathbf{R}^{2}\right)$. Q.E.D. 
We now relate the fixed points of the operator $T$ to the solutions of equations (4), (7).

Proposition 5. For any $(u, v) \in C(\bar{\Omega}) \times C(\bar{\Omega})$ and $s \in[0,1]$ then $(u, v)=$ $s T(u, v)$ if and only if $(u, v)$ is a solution of the equations

$$
\begin{aligned}
& \nabla^{2} u+\lambda g f(u, v)=0 \quad \text { in } \Omega, \quad \partial_{u} u / \partial n+\mu u=0 \text { on } \partial \Omega, \\
& \nabla^{2} v-\alpha \lambda s f(u, v)=0 \quad \text { in } \Omega, \quad \partial v / \partial n+\nu v=0 \quad \text { on } \partial \Omega .
\end{aligned}
$$

Proof. Suppose $(u, v) \in C(\bar{\Omega}) \times C(\bar{\Omega})$ satisfies $(u, v)=s T(u, v)$. Then

$$
u=\lambda s K_{\mu} F(u, v)
$$

and

$$
v=-\lambda \alpha s K_{\nu} F(u, v)
$$

Now, $u, v \in C(\bar{\Omega})$ and $f \in C^{1}\left(\mathbf{R}^{2}\right)$, so $F(u, v) \in C(\bar{\Omega})$. Then, by Proposition 1 , $K_{\mu} F(u, v), K_{\nu} F(u, v) \in C^{\sigma}(\bar{\Omega})$; so $u, v \in C^{\sigma}(\bar{\Omega})$.

Therefore $F(u, v) \in C^{\sigma}(\bar{\Omega})$, which gives, by Proposition 1, $K_{\mu} F(u, v)$, $K_{\nu} F(u, v) \in C^{2+\sigma}(\bar{\Omega})$. Hence $u, v \in C^{2+\sigma}(\bar{\Omega})$. Now it follows from the definition of $K_{\mu}$ and $K_{\nu}$ (Proposition 1) that $(u, v)$ satisfy (12).

The converse follows trivially from the definition of $K_{\mu}$ and $K_{\nu}$. Q.E.D.

We note here that Propositions 4 and 5 hold for the case $\mu<\nu=\infty$. We shall later have need of this fact.

We now proceed to show that $T$ does indeed have fixed points. Our first step is to define a homotopy between $I$ and $I-T$ satisfying Proposition 2(iii). We do this as follows:

$$
\begin{aligned}
& \text { for } s \in[0,1] \text { and }(u, v) \in C(\bar{\Omega}) \times C(\bar{\Omega}), \\
& (u, v)-H((u, v), s)=(u, v)-s T(u, v)
\end{aligned}
$$

In order to show that $H$ satisfies Proposition 2(iii) we need:

LEMMA 6. If, for $s \in[0,1]$ and $(u, v) \in C(\bar{\Omega}) \times C(\bar{\Omega})$,

$$
(u, v)=s T(u, v)
$$

then $(\forall x \in \bar{\Omega}) v_{0} \leqslant v(x) \leqslant 0$, and

$$
\begin{aligned}
& 0 \leqslant u(x) \leqslant-v_{0} / \alpha, \text { for } \nu \leqslant \mu, \\
& 0 \leqslant u(x) \leqslant-v_{0} \nu / \alpha \mu, \text { for } \mu<\nu<\infty,
\end{aligned}
$$

where $v_{0}$ is given in Section 1 (iii). 
Proof. First, by Proposition $5,(u, v)$ satisfies (12). Suppose now that $v-v_{0}$ achieves a negative minimum at $x^{\prime} \in \Omega$; then there exists an open set $\Omega_{0} \subseteq \Omega$ such that $x^{\prime} \in \Omega_{0}$ and $v<v_{0}$ on $\Omega_{0}$. It then follows from the properties of $f((\mathrm{i})$ and (ii) in Section 1) and (12b) that

$$
\nabla^{2}\left(v-v_{0}\right)=\lambda \alpha s f(u, v)<0, \quad \text { in } \Omega_{0} .
$$

Consequently, by the maximum principle $v-v_{0}$ is constant on $\Omega_{0}$, which contradicts (13). The boundary condition in (12b) ensures that $v-v_{0}$ does not achieve a negative minimum on $\partial \Omega$. So $(\forall x \in \bar{\Omega}) v_{0} \leqslant v(x)$.

From this it follows that

$$
\begin{array}{ll}
0=\nabla^{2} v-\lambda \alpha s f(u, v) \leqslant \nabla^{2} v & \text { in } \Omega, \\
\partial v / \partial n+v v=0 & \text { on } \partial \Omega .
\end{array}
$$

Again, applying the maximum principle shows that

$$
(\forall x \in \bar{\Omega}) \quad v(x) \leqslant 0 .
$$

Now, it follows from (12) that ( $v, h=\alpha u+v$ ) satisfies (10) (with $\lambda$ replaced by $\lambda s)$. Then, using the above bounds for $v$, we get

$$
\begin{aligned}
& \nabla^{2} h=0, \quad \text { in } \Omega, \\
& \partial h / \partial n+\mu h=(\mu-\nu) v\left\{\begin{array}{l}
\leqslant 0 \\
\geqslant(\mu-\nu) v_{0}
\end{array} \quad \text { if } \mu \geqslant \nu,\right. \\
& \partial h / \partial n+\mu h=(\mu-\nu) v\left\{\begin{array}{l}
\geqslant 0 \\
\leqslant(\mu-\nu) v_{0}
\end{array} \quad \text { if } \mu<\nu .\right.
\end{aligned}
$$

The maximum principle then show that

$$
\begin{array}{lll}
(\forall x \in \bar{\Omega}) & (1-\nu / \mu) v_{0} \leqslant h(x) \leqslant 0 & \text { for } \mu \geqslant \nu, \\
& 0 \leqslant h(x) \leqslant(1-\nu / \mu) v_{0} & \text { for } \nu>\mu
\end{array}
$$

(if $\mu=\nu=\infty$ we note that $h=0$ ).

Since $u=\alpha^{-1}(h-v)$, we then get (where we also apply the maximum principle to $(12 a))$

$$
\begin{aligned}
& (\forall x \in \bar{\Omega}) \quad 0 \leqslant u(x) \leqslant-v_{0} / \alpha \quad \text { for } \mu \geqslant \nu, \\
& 0 \leqslant u(x) \leqslant-\nu v_{0} / \alpha \mu \quad \text { for } \mu<\nu<\infty . \quad \text { Q.E.D. }
\end{aligned}
$$

For convenience we denote

$$
m(\alpha)= \begin{cases}-v_{0} / \alpha & \text { for } \mu \geqslant \nu \\ -\nu v_{0} / \alpha \mu & \text { for } \mu<\nu\end{cases}
$$

We are now in a position to prove:

THEOREM 7. There exists $(u, v) \in C(\bar{\Omega}) \times C(\bar{\Omega})$ such that $(u, v)=T(u, v)$. 
Proof. Let $B=\left\{(u, v) \in C(\bar{\Omega}) \times C(\bar{\Omega}):\|u\|_{0}+\|v\|_{0}<1-v_{0}+m(\alpha)\right\}$, and $H$ be as defined after Proposition 5. By Proposition 4 it is easily seen that, for each $s \in[0,1], H_{s}=H(\cdot, s)$ is a compact map from $\bar{B}$ into $C(\bar{\Omega}) \times C(\bar{\Omega})$. Since $T$ is compact, it maps $\bar{B}$ into a relatively compact set, and therefore into a bounded set. So, for $(u, v) \in \bar{B}$ and $s_{1}, s_{2} \in[0,1]$,

$$
\left\|H\left((u, v), s_{1}\right)-H\left((u, v), s_{2}\right)\right\|_{0}=\left|s_{1}-s_{2}\right|\|T(u, v)\|_{0} \leqslant \Gamma\left|s_{1}-s_{2}\right|
$$

for some constant $\Gamma$.

From Lemma 6, $0 \notin\left(I-H_{s}\right)(\partial B)$ for each $s \in[0,1]$. Thus the conditions of Propositon 2(iii) are satisfied. Then, by Proposition 2(i) and (iii),

$$
d(I-T, B, 0)=d(I, B, 0)=1 .
$$

So, by Proposition 2(ii), there is some $(u, v) \in B$ such that

$$
(u, v)=T(u, v) . \quad \text { Q.E.D. }
$$

COROLlaRY 8. There exists a solution $(u, v)$ to $(4),(7)$.

This is a consequence of Proposition 5 and Theorem 7.

COROLlaRY 9. (Of the proof)

$$
d(I-T, B, 0)=1 .
$$

In our method for showing the existence of solutions to (4), (7) it has been of importance to derive an a priori estimate for the solution $h=\alpha u+v$ of $10(\mathrm{~b})$, namely

$$
\begin{array}{lll}
(1-\nu / \mu) v_{0} \leqslant h \leqslant 0 & \text { in } \bar{\Omega}, & \text { for } \mu \geqslant \nu, \\
(1-\nu / \mu) v_{0} \geqslant h \geqslant 0 & \text { in } \bar{\Omega}, & \text { for } \mu<\nu<\infty .
\end{array}
$$

For the parameter range not covered in this section, viz. $\mu<\nu=\infty$, we no longer have such a priori bounds for $k$. This is the reason that the argument given in this section fails for this case. It transpires that there may be no solution for certain values of $\lambda$.

\section{Non-existence and existence of solutions for $\mu<\nu=\infty$}

In this section we shall investigate the question of existence of solutions to (4), (5), equivalently (10), for the special case of $\nu=\infty$ with $\mu$ finite. Of course, we continue to examine the general case of $\alpha>0$.

In line with Section 2 we shall generally use formulation (10) of the problem (4), (5). However, we firstly obtain a regularity property of a solution $(u, v)$ to (4), (7). 
Lemma 1. Suppose $(U, V)$ satisfies (4), (7) for some $\lambda$; then $V \in C^{2+o}(\bar{\Omega})$.

Proof. Firstly, note that since $U \in C^{1}(\bar{\Omega}), U \in C^{\sigma}(\bar{\Omega})$. From the maximum principle, it follows that the equation

$$
\left.\begin{array}{rl}
\nabla^{2} w-\alpha \lambda F(u, w)=0 & \text { in } \Omega, \\
w=0 & \text { on } \partial \Omega,
\end{array}\right\}
$$

has a unique solution for specified $u \in C^{\sigma}(\bar{\Omega})$. We now show that there is a solution, $w$, to (14) which is in $C^{2+\sigma}(\bar{\Omega})$. To do this we define a map, for fixed $u \in C^{\sigma}(\bar{\Omega})$,

$$
S: C(\bar{\Omega}) \rightarrow C(\bar{\Omega}): w \mapsto \alpha \lambda K_{\infty} F(u, w),
$$

where $K_{\infty}$ is the operator defined in Section 2, Proposition 1, when $\nu=\infty$.

Using similar arguments to those in Section 2, Propositions 4, 5 and Theorem 7, we can show that: $S$ is well-defined and compact in the supremum norm; if $w \in C(\bar{\Omega})$ satisfies $w=S w$ then it also satisfies (14); if $w=s S w$, for $s \in[0,1]$ and $w \in C(\bar{\Omega})$, then $\|w\|_{0} \leqslant-v_{0}$; the map $H:\left\{w:\|w\|_{0}<1-v_{0}\right\} \times[0,1] \rightarrow$ $C(\bar{\Omega}):(w, s) \mapsto s S w$ satisfies the conditions of Section 2, Proposition 2(iii). Consequently there exists some $w \in C^{2+\sigma}(\bar{\Omega})$ such that $w$ satisfies (14). Since the solution to (14) is unique when $u=U$ and $(U, V)$ satisfies (4), (7), it follows that $V$ satisfies (14) and so $V \in C^{2+o}(\bar{\Omega})$. Q.E.D.

The following argument requires us to find an explicit solution to $\nabla^{2} v+\theta v=0$ in a sphere, with spherical symmetry. So the result (Theorem 2) is restricted to the cases $n=1,2,3$. We give the proof for $n=3$, and note that the result for $n=1,2$ can be derived by imbedding the region in 3-dimensions.

Suppose now, that, for some value $\lambda$, there is a solution $(u, v)$ to (4), (5), and hence a solution $(v, h)$ to $(10)$. Then Lemma 1 allows us to define the quantity

$$
M=\min \left\{\frac{\partial v}{\partial n}(x): x \in \partial \Omega\right\} .
$$

Since $v \leqslant 0$ in the region $\Omega$, it follows that $M \geqslant 0$. We now proceed to establish the following inequality:

$$
M \geqslant\left(\alpha \lambda e^{M / \alpha \mu}\right)^{1 / 2} \operatorname{coth}\left[\left(\alpha \lambda e^{M / \alpha \mu}\right)^{1 / 2} \rho\right]-1 / \rho,
$$

(see below for the definition of $\rho$ ).

Now, from (10), we have

$$
\begin{aligned}
& \nabla^{2}(h-M / \mu)=0 \quad \text { in } \Omega, \\
& \frac{\partial}{\partial n}(h-M / \mu)+\mu(h-M / \mu)=\frac{\partial v}{\partial n}-M \geqslant 0 \quad \text { on } \partial \Omega .
\end{aligned}
$$

Therefore, by the maximum principle, $h \geqslant M / \mu$ in $\bar{\Omega}$. 
Since $\partial \Omega$ is of class $C^{2+\sigma}$, it satisfies a uniform interior sphere condition, that is, there exists $\rho>0$ such that, for each $x \in \partial \Omega$, there is an open ball of radius $\rho$, $B_{x}$, such that $B_{x} \subseteq \Omega$ and $x \in \partial B_{x} \cap \partial \Omega$.

Now, fix $x_{0} \in \partial \Omega$ and let $\Omega_{0}=B_{x_{0}}$. We define a function $v_{1}$, on $\Omega_{0}$, to be the unique solution of

$$
\left.\begin{array}{rl}
\nabla^{2} w-\alpha \lambda(1+w) e^{M / \alpha \mu}=0 & \text { in } \Omega_{0}, \\
w=0 & \text { on } \partial \widehat{\Omega}_{0} .
\end{array}\right\}
$$

Also, we have

$$
\begin{aligned}
\nabla^{2} v-\alpha \lambda(1+v) e^{M / \alpha \mu} & =\alpha \lambda(1+v)\left[e^{(h-v) / \alpha}-e^{M / \alpha \mu}\right] \\
& \geqslant 0 \text { in } \Omega_{0},
\end{aligned}
$$

and

$$
v \leqslant 0 \text { on } \partial \Omega_{0},
$$

since $h \geqslant M / \mu$ and $v \leqslant 0$. Consequently $v$ is a lower solution of (16). As 0 is an upper solution of (16), it follows that

$$
\left(\forall x \in \Omega_{0}\right) \quad v(x) \leqslant v_{1}(x) \leqslant 0 .
$$

Moreover,

$$
v_{1}=\rho \sinh \left[\left(\alpha \lambda e^{M / \alpha \mu}\right)^{1 / 2} r\right] /\left(r \sinh \left[\left(\alpha \lambda e^{M / \alpha \mu}\right)^{1 / 2} \rho\right]\right)-1,
$$

where $r$ is the distance from the centre of $B_{x_{0}}$. Since $v\left(x_{0}\right)=v_{1}\left(x_{0}\right)=0$, it follows from (17) that

$$
\begin{aligned}
\frac{\partial v}{\partial n}\left(x_{0}\right) & \geqslant \frac{\partial v_{1}}{\partial n}\left(x_{0}\right)=\left.\frac{\partial v_{1}}{\partial r}\right|_{r=\rho} \\
& =\left(\alpha \lambda e^{M / \alpha \mu}\right)^{1 / 2} \operatorname{coth}\left[\left(\alpha \lambda e^{M / \alpha \mu}\right)^{1 / 2} \rho\right]-1 / \rho .
\end{aligned}
$$

Taking $x_{0}$ to be the point on $\partial \Omega$ at which $\partial v / \partial n$ achieves its minimum, (15) then follows. Since $\operatorname{coth} \theta \geqslant 1$ for $\theta \geqslant 0$, we obtain

$$
\lambda \leqslant \alpha^{-1}\left(M+\rho^{-1}\right)^{2} e^{-M / \alpha \mu} .
$$

By finding.the maximum of the right hand side of this inequality, as a function of $M$, we have

$$
\lambda \leqslant 4 \alpha \mu^{2} e^{-2+1 / \rho \alpha}
$$

This last.inequality leads to the result:

THEOREM 2. For $\lambda$ sufficiently large, in particular $\lambda>4 \alpha \mu^{2} e^{-2+1 / \rho \alpha \mu}$, there is no solution to (4), (5). 
A similar result may be obtained for (4), (7) if $e^{u}$ is replaced by a function of $u$ that grows faster than $u^{2}$ as $u$ tends to infinity.

Next we shall show that (4), (7) does have a solution for some values of $\lambda$-in particular, when $\lambda$ is sufficiently small. Again we consider a map $T$ defined on $C(\bar{\Omega}) \times C(\bar{\Omega})$ by

$$
T(u, v)=\left(\lambda K_{\mu} F(u, v),-\alpha \lambda K_{\infty} F(u, v)\right),
$$

where $K_{\mu}, K_{\infty}$ are as in Section 2, Proposition 1. As noted after Section 2, Proposition 5, we have:

LEMMA 3. The mapping $T:\left(C(\bar{\Omega}) \times C(\bar{\Omega}),\|\|_{0} \times\|\|_{0}\right) \rightarrow\left(C(\bar{\Omega}) \times C(\bar{\Omega}),\|\|_{0}\right.$ $\left.\times\|\|_{0}\right)$ is well-defined and continuous.

We also have:

Proposition 4. Let $B=\left\{(u, v) \in C(\bar{\Omega}) \times C(\bar{\Omega}):\|u\|_{0}+\|v\|_{0}<r\right\}$ for some $r>0$; then, for sufficiently small $\lambda, T$ is a contraction map from $\bar{B}$ to $\bar{B}$.

Proof. From the continuity of $f$, we have, for $(u, v) \in \bar{B}$,

$$
\|F(u, v)\|_{0} \leqslant M
$$

for some positive $M$. Since $f \in C^{1}\left(\mathbf{R}^{2}\right)$, it follows that there is a constant $C>0$, such that for $(u, v),\left(u_{1}, v_{1}\right) \in \bar{B}$,

$$
\left\|F(u, v)-F\left(u_{1}, v_{1}\right)\right\|_{0} \leqslant C\left\|(u, v)-\left(u_{1}, v_{1}\right)\right\|_{0} .
$$

Hence

$$
\left\|T(u, v)-T\left(u_{1}, v_{1}\right)\right\|_{0} \leqslant \lambda\left(\left\|K_{\mu}\right\|_{0}+\alpha\left\|K_{\infty}\right\|_{0}\right) C\left\|(u, v)-\left(u_{1}, \widehat{v_{1}}\right)\right\|_{0}
$$

where $\left\|K_{\mu}\right\|_{0}$ and $\left\|K_{\infty}\right\|_{0}$ are the norms of the operators $K_{\mu}$ and $K_{\infty}$. Also, for $(u, v) \in \bar{B}$,

$$
\|T(u, v)\|_{0} \leqslant \lambda\left(\left\|K_{\mu}\right\|_{0}+\alpha\left\|K_{\infty}\right\|_{0}\right) M .
$$

Consequently, for $\lambda$ sufficiently small, $T$ is a contraction map from $\bar{B}$ to $\bar{B}$. Q.E.D.

This result then leads to:

THEOREM 5. For $\lambda$ sufficiently small, (4), (7) has a solution.

Proof. Choose some $r>0$; then by Proposition $4, T$ is a contraction map from $\bar{B}$ to $\bar{B}$ for $\lambda$ sufficiently small. $\bar{B}$ is a closed subset of $C(\bar{\Omega}) \times C(\bar{\Omega})$, hence it is 
complete. It then follows from the contraction mapping theorem that $T$ has a unique fixed point $(u, v) \in \bar{B}$. Therefore, by the note after Section 2, Proposition $5,(u, v)$ is a solution to (4), (7). Q.E.D.

We have now shown that for $\lambda$ sufficiently small (4), (5) has a solution, but for $\lambda$ sufficiently large there is no solution to (4), (5). In fact we have a stronger result which we give after the following theorem:

THEOREM 6. If there is a solution $\left(u^{0}, v^{0}\right)$ to (4), (7) for $\lambda=\lambda^{0}$ then there is a solution to (4), (7) for each $\lambda \in\left[0, \lambda^{0}\right]$.

Proof. Since $\left(u^{0}, v^{0}\right)$ is a solution to (4), (7), then (11) has a solution $\left(u^{0}, h^{0}\right)$ when $\lambda=\lambda^{0}$. We then consider, for $\lambda<\lambda^{0}$, the iteration scheme:

$$
h_{0}=0 \text { in } \Omega \text {; }
$$

for $j>0$,

$$
\left.\begin{array}{ll}
\nabla^{2} u_{j}+\lambda f\left(u_{j}, h_{j-1}-\alpha u_{J}\right)=0 & \text { in } \Omega \\
\frac{\partial u_{j}}{\partial n}+\mu u_{J}=0 & \text { on } \partial \Omega
\end{array}\right\}
$$

and

$$
\left.\begin{array}{ll}
\nabla^{2} h_{j}=0 & \text { in } \Omega \\
h_{j}=\alpha u_{j} & \text { on } \partial \Omega
\end{array}\right\}
$$

where $u$, is the minimal solution to (19a) (which is known to exist by the work in Keller and Cohen [6]).

If $h_{j}>h_{j-1}$ in $\Omega$ then, using the monotonicity of $f$,

$$
\begin{aligned}
\nabla^{2} u_{j+1}+\lambda f\left(u_{j+1},\right. & \left.h_{J-1}-\alpha u_{j+1}\right) \\
& =\lambda\left(f\left(u_{\jmath+1}, h_{j-1}-\alpha u_{j+1}\right)-f\left(u_{j+1}, h_{\jmath}-\alpha u_{j+1}\right)\right) \leqslant 0 .
\end{aligned}
$$

Hence $u_{\jmath+1}$ is an upper solution for the equation satisfied by $u_{j}$; also 0 is a lower solution, so $u_{\jmath+1} \geqslant u_{\jmath}$. It then follows that $h_{\jmath+1} \geqslant h_{\jmath}$.

Now, $u_{1} \geqslant 0$, hence $h_{1} \geqslant 0$. Therefore it follows inductively that $u$, and $h_{\text {, are }}$ monotone increasing in $j$.

Moreover, if $h_{j-1} \leqslant h^{0}$, $\nabla^{2} u^{0}+\lambda f\left(u^{0}, h_{j-1}-\alpha u^{0}\right)=\lambda f\left(u^{0}, h_{j-1}-\alpha u^{0}\right)-\lambda^{0} f\left(u^{0}, h^{0}-\alpha u^{0}\right) \leqslant 0$.

So $u^{0}$ is an upper solution for the equation satisfied by $u_{j}$. Therefore $u_{j} \leqslant u^{0}$, and so $h_{j} \leqslant h^{0}$. Since $h_{0}=0$, it also follows from an inductive argument that, for each $j, u_{j}$ and $h_{j}$ are bounded above by $u^{0}$ and $h^{0}$ respectively. 
As in Keller and Cohen [6] we can then deduce that there is a solution $(u, h)$ to (11), for any $\lambda \leqslant \lambda^{0}$, such that

$$
0 \leqslant u \leqslant u^{0} \text { in } \Omega
$$

and

$$
0 \leqslant h \leqslant h^{0} \text { in } \Omega .
$$

(This is done by using the arguments of Keller and Cohen [6] for one equation to show that $\left(\lim _{j \rightarrow \infty} u_{j}, \lim _{j \rightarrow \infty} h_{j}\right)$ is a solution to (11).) Q.E.D.

COROLlaRy 7. There exists a number $\lambda^{*}$ such that (4), (5) has a solution for $\lambda<\lambda^{*}$, and no solution for $\lambda>\lambda^{*}$.

Proof. Corollary 7 follows from Theorems 2 and 6.

The question as to whether or not there is a solution when $\lambda=\lambda^{*}$ is as yet unresolved. For the one-dimensional case we have determined that there is indeed a solution for $\lambda=\lambda^{*}$ (for the details see the second part of this paper).

\section{Notes on Section 3}

1) The results Lemma 3, Proposition 4 and Theorems 5 and 6 all hold in arbitrary dimensions (not just $n \leqslant 3$ ); the proofs for the general case being the same as those presented here.

2) The monotone iteration scheme in (19) can be used to show that solutions to (4), (7) exist if $\mu<\nu<\infty$ and $\lambda$ is arbitrary (the boundary condition in (19b) needs to be changed to $\left.\partial h_{j} / \partial n+\nu h_{j}=\alpha(\nu-\mu) u_{j}\right)$. In this case one shows that the sequences $u$, and $h$, are bounded above by $-v_{0} \nu / \alpha \mu$ and $-v_{0}(\nu / \mu-1)$ respectively. Moreover, as in Keller and Cohen [6] this monotone scheme can be used to show the existence of a minimal solution pair $(u, h)$ to (11), and a maximal solution pair $(U, H)$ to (11).

This concludes our discussion of the existence of solutions to (4), (7). We now turn our attention to the behaviour of any solutions as the parameter $\lambda$ varies.

\section{Continuity of solutions with respect to $\lambda$}

In this section we shall examine some of the properties of the set of solutions to (4), (7). Ideally we would like to show that any two solutions to (4), (7) can be joined by a path (in $C(\bar{\Omega}) \times C(\bar{\Omega}) \times \mathbf{R}^{+} \cup\{0\}$ ) of solutions. 
The results and proofs that we shall give are of a standard type; but for the sake of completeness we present them here.

For the first two results we can assume $0<\mu, \nu \leqslant \infty$. Let

$$
\begin{aligned}
& X=C(\bar{\Omega}) \times C(\bar{\Omega}) \times\left(\mathbf{R}^{+} \cup\{0\}\right), \\
& \Sigma=\left\{\left((u, v), \lambda_{1}\right):(u, v) \text { is a solution to }(4),(7) \text { with } \lambda=\lambda_{1}\right\},
\end{aligned}
$$

and

$$
Q= \begin{cases}T / \lambda & \text { for } \lambda>0 \\ 0 & \text { for } \lambda=0\end{cases}
$$

where $T$ is the map given in Section $2(\nu<\infty)$ or $(18)(\nu=\infty)$. First we need the following result:

LEMMA $1 . \Sigma$ is a closed subset of $X$, and further, if $U$ is a closed bounded subset of $X$ then $\Sigma \cap U$ is a compact set.

Proof. From Section 2, Proposition 5, we see that

$$
\Sigma=\{((u, v), \lambda):(u, v)=\lambda Q(u, v)\} .
$$

The lemma now follows from the continuity and compactness of $Q$ (Section 2, Proposition 4). Q.E.D.

We then get the general result:

THEOREM 2. $\Sigma$ contains an unbounded subcontinuum, $\Gamma$, such that $((0,0), 0) \in \Gamma$.

Proof. Firstly we note that a continuum is a closed connected set. So, if no such $\Gamma$ exists then the component of $\Sigma$ containing $((0,0), 0), C$ say, is bounded. Hence, there exists $r>0$ such that

$$
C \subset B(r)=\left\{((u, v), \lambda) \in X:\|(u, v)\|_{0}<r \text { and } \lambda<r\right\} .
$$

Consequently, by Lemma $1, C$ and $D=(\partial B(r)) \cap \Sigma$ are closed disjoint subsets of the compact metric space $\overline{B(r)} \cap \Sigma$. Since $C$ is a component and $D$ is compact, it follows from a result in Engelking [3] (6.123) that there exist disjoint compact sets $E_{1}, E_{2}$ such that

$$
C \subseteq E_{1}, \quad D \subseteq E_{2} \text { and } E_{1} \cup E_{2}=\overline{B(r)} \cap \Sigma .
$$

As $\Sigma$ is closed, $E_{1}$ and $E_{2}$ are closed disjoint subsets of $\overline{B(r)}$. So there exists an open set $U \subset \overline{B(r)}$ such that

$$
E_{1} \subseteq U \text { and } \bar{U} \cap E_{2}=\varnothing .
$$

Therefore $\partial U \cap \Sigma=\varnothing$. 
Using (20), we then have that, for $((u, v), \lambda) \in \partial U$,

$$
(u, v) \neq \lambda Q(u, v) \text {. }
$$

Hence, by Section 2, Proposition 2(iv),

$$
d\left(I-\lambda Q, U_{\lambda}, 0\right) \text { is independent } \mathrm{f} \lambda \text { on }[0,2 r],
$$

where $U_{\lambda}=\{(u, v):((u, v), \lambda) \in U\}$.

Now $U_{2 r}=\varnothing$, so by Section 2, Proposition 2(ii),

$$
d\left(I-\lambda Q, U_{2 r}, 0\right)=0 .
$$

Also, $(0,0) \in U_{0}$ (as $C \subseteq U$ ), so, by Section 2, Proposition 2(i),

$$
d\left(I-0 . Q, U_{0}, 0\right)=d\left(I, U_{0}, 0\right)=1 .
$$

But this contradicts (21), so the result follows. Q.E.D.

Theorem 2 does not tell us that any two points in $\Sigma$ (or even $\Gamma$ ) can be joined by a path lying in $\Sigma$ (that is a path of solutions to (4), (7)); but it does tell us that there is an unbounded subset of solutions, $\Gamma$, which contains the trivial solution $u=0, v=0$ and $\lambda=0$ and for which it is impossible to find a ball in $X$ of radius $r$, about the origin, which contains no elements of $\Gamma$ on its boundary.

We are able to give a stronger result for a certain special case. Since we require Section 2, Corollary 9 we need to assume that $\mu, \nu<\infty ; \nu<\infty$ and $\mu \leqslant \infty$ or $\mu=\nu=\infty$.

THeOREM 3. Suppose $J$ is an open (not necessarily bounded) interval in $\mathbf{R}^{+} \cup\{0\}$ such that, for each $\lambda \in J$, there is only one solution $(u(\lambda), v(\lambda))$ to (4), (7). Then the function $J \rightarrow C(\bar{\Omega}) \times C(\bar{\Omega}): \lambda \mapsto(u(\lambda), v(\lambda))$ is continuous.

Proof. Suppose the function is discontinuous at $\lambda_{0} \in J$. Then there exists $\varepsilon_{0}>0$ such that

$$
(\forall n)\left(\exists \lambda_{n} \in J\right) \text { so that }\left|\lambda_{n}-\lambda_{0}\right|<1 / n
$$

and

$$
\left\|\left(u\left(\lambda_{n}\right), v\left(\lambda_{n}\right)\right)-\left(u\left(\lambda_{0}\right), v\left(\lambda_{0}\right)\right)\right\|_{0}>\varepsilon_{0} .
$$

Now, by Section 2, Proposition 3(ii), there exists $r_{0}>0$ such that whenever $\left|\lambda-\lambda_{0}\right|<r_{0}$,

$d\left(I-\lambda_{0} Q, B\left(\left(u\left(\lambda_{0}\right), v\left(\lambda_{0}\right)\right) ; \varepsilon_{0}\right), 0\right)=d\left(I-\lambda Q, B\left(\left(u\left(\lambda_{0}\right), v\left(\lambda_{0}\right)\right) ; \varepsilon_{0}\right), 0\right)$ 
where $B((U, V) ; r)=\left\{(u, v) \in C(\bar{\Omega}) \times C(\bar{\Omega}):\|U-u\|_{0}+\|V-v\|_{0}<r\right\}$. This follows since there is only one solution to (4), (7) when $\lambda=\lambda_{0}$ and so, by Section 2, Proposition 5, there is only one fixed point of the map $\lambda_{0} Q$.

Also, by Section 2, Corollary 9 and Section 2, Proposition 3(i),

$$
\left.\begin{array}{rl}
1 & =d\left(I-\lambda_{0} Q, B\left((0,0) ; 1-v_{0}+m(\alpha)\right), 0\right) \\
& =d\left(I-\lambda_{0} Q, B\left(\left(u\left(\lambda_{0}\right), v\left(\lambda_{0}\right)\right) ; \varepsilon_{0}\right), 0\right) .
\end{array}\right\}
$$

On the other hand, since $\left(u\left(\lambda_{n}\right), v\left(\lambda_{n}\right)\right) \notin B\left(\left(u\left(\lambda_{0}\right), v\left(\lambda_{0}\right)\right) ; \varepsilon_{0}\right)$, it follows from Section 2, Proposition 2(ii), that for $1 / n<r_{0}$,

$$
d\left(I-\lambda_{n} Q, B\left(\left(u\left(\lambda_{0}\right), v\left(\lambda_{0}\right)\right) ; \varepsilon_{0}\right), 0\right)=0 .
$$

But this contradicts (22); so the theorem must hold. Q.E.D.

This result tells us that two solutions corresponding to values of $\lambda$ in $J$ can indeed be connected by a path (in $X$ ) of solutions. In the second part of this paper we shall show that it is possible to find such an interval $J$.

\section{Discussion}

In this paper we have shown that, except for the extreme case of $\mu<\nu=\infty$, there is a solution to the steady state system (4), (7) for all values of $\lambda$. This suggests that other than for this special case, a solution to the initial value problem (3), (4), with $u$ sufficiently small and $v<0$ at $t=0$, remains bounded and we do not obtain thermal runaway. It is plausible that this solution $(u, v)$ either approaches one of the steady states (that is solutions to (4), (5)) or tends to a limit cycle ( $c f$. Poore [10] for the corresponding "zero-dimensional case"-that is the homogeneous system).

For the special case of $\mu\langle\nu=\infty$ in 1, 2 or 3 dimensions, we see that there is some $\lambda^{*}$ such that there is no solution to (4), (5) for $\lambda>\lambda^{*}$, that is there is no steady solution for the parabolic problem (3), (4) if the region $\Omega$ is too large (since $\lambda \propto(\text { radius of } \Omega)^{2}$ ). It can be seen this parameter range corresponds to free transfer of reactant through the surface $\partial \Omega$ into $\Omega$, while the flow of heat out of $\Omega$ through $\partial \Omega$ is restricted (however, this seems to be a rather unrealistic case). Hence, it would appear the physical reason that there is no solution for large values of $\lambda$ is that heat is produced from the incoming reactant too rapidly to be lost through the surface. It then seems plausible that if $\lambda>\lambda^{*}$ then the solution to (3), (4) blows-up, with $u$ becoming infinite in either infinite or finite time ( $c f$. the case $\alpha=0, v \equiv 0$ Fujita [4], Fujita [5], Lacey [7]). 


\section{References}

[1] H. Amann, "Fixed point equations and non-linear eigenvalue problems in ordered Banach spaces", SIAM Rev. 18 (1976), 620-709.

[2] R. Aris, The mathematical theory of diffusion and reaction in permeable catalysts, Vol. 1 (Oxford University Press, 1975).

[3] R. Engelking, General topology (Polish Scientific Publishers, Warsaw, 1975).

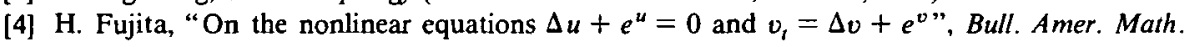
Soc. 75 (1969), 132-135.

[5] H. Fujita, "On some nonexistence and nonuniqueness theorems for nonlinear parabolic equations", Proc. of Symposia in Pure Math. 28 Part I, Amer. Math. Soc. (1970), 105-113.

[6] H. B. Keller and D. S. Cohen, "Some positone problems suggested by nonlinear heat generation", J. Math. Mech. 16 (1967), 1361-1376.

[7] A. A. Lacey, "Mathematical analysis of thermal runaway for spatially inhomogeneous reactions", SIAM J. Appl. Math. (in press).

[8] O. A. Ladyzhenskaya and N. N. Ural'tseva, Linear and quasilinear elliptic equations (Academic Press, New York, 1968).

[9] N. G. Lloyd, Degree theory (Cambridge University Press, 1978).

[10] A. B. Poore, "Stability and bifurcation phenomena in chemical reactor theory", Arch. Rational Mech. Anal. 52 (1973), 358-388.

[11] D. H. Sattunger, "Topics in stability and bifurcation theory", Lecture Notes in Mathematics 309 (Springer-Verlag, New York, 1973). 